\title{
Mo-Based Proximity Bilayers for TES: Microstructure and Properties
}

\author{
Lourdes Fàbrega, Iván Fernández-Martínez, Óscar Gil, María Parra-Borderías, Agustín Camón, \\ José-Luis Costa-Krämer, Raquel González-Arrabal, Javier Sesé, Fernando Briones, José Santiso, and
} Francesca Peiró

\begin{abstract}
We report on the fabrication and characterization of Mo films, Mo/Au and Mo/Cu bilayers for Transition Edge Sensors (TES). The fabrication conditions (at room temperature) have been varied to achieve layers with the required properties for TES applications. The dependence of their functional properties (i.e. electrical resistivity and superconducting critical temperature) on microstructure (grain size, stress) is investigated.
\end{abstract}

Index Terms-Proximity effect, radiation detectors, stress, transition edge sensors.

\section{INTRODUCTION}

$\mathbf{T}$ RANSITION edge sensors (TES), based on superconductors, can constitute microcalorimeters with excellent performances as photon detectors with spectroscopic capabilities. Among other applications, they are considered at present as the most promising option for X-ray imaging spectrometers in the next generation of X-ray astronomical missions [1], [2].

TES are usually bilayers constituted by a superconductor (S) and a normal conducting metal $(\mathrm{N})$ layer: the proximity effect between both layers [3] allows an excellent tuning of the superconducting critical temperature $T_{c}$, which determines the operating point of the detector (usually $\sim 100 \mathrm{mK}$ ), while maintaining very narrow transitions, which are essential for high spectral energy resolution. $T_{C}$ is mainly governed by the critical temperature of the $\mathrm{S}$ layer, the thicknesses and electronic properties of the $\mathrm{S}$ and $\mathrm{N}$ layers and the interface between them.

Manuscript received August 18, 2008. Current version published July 10, 2009. This work was supported by the Spanish Ministerio de Educación y Ciencia, MEC (Projects MAT2005-02454 and ESP2006-13608-C02).

L. Fàbrega and Ó Gil are with the Institut de Ciència de Materials de Barcelona (CSIC), 08193 Bellaterra, Spain (e-mail: lourdes@icmab.es; ogil@icmab.es).

I. Fernández-Martínez, J.-L. Costa-Krämer, R. González-Arrabal, and F. Briones are with the Instituto de Microelectrónica de Madrid (CSIC), 28760 Tres Cantos, Spain (e-mail: ivan@imm.cnm.csic.es; kramer@imm.cnm.csic.es; arrabal@imm.cnm.csic.es; briones@imm.cnm.csic.es).

M. Parra-Borderías and A. Camón are with the Instituto de Ciencia de Materiales de Aragón (CSIC), Zaragoza, Spain (e-mail: mariapb@unizar.es; acamon@unizar.es).

J. Sesé is with the Instituto de Nanociencia de Aragón, Universidad de Zaragoza, Zaragoza, Spain (e-mail: jsese@unizar.es).

J. Santiso is with the Institut de Nanociencia i Nanotecnologia CIN2 (CSICICN), Bellaterra, Spain (e-mail: jsantiso@icmab.es).

F. Peiró is with the Dpt. d'Electrònica, Universitat de Barcelona, Barcelona, Spain (e-mail: fpeiro@ub.edu).

Color versions of one or more of the figures in this paper are available online at http://ieeexplore.ieee.org.

Digital Object Identifier 10.1109/TASC.2009.2019052
Therefore, a crucial aspect is an excellent control of the fabrication process, which will determine the microstructure of each layer (and therefore its electric properties) and the quality of the $\mathrm{S} / \mathrm{N}$ interface.

The most studied and with best spectral resolution TES have $\mathrm{Ti}$ or $\mathrm{Mo}$ as superconductor, and $\mathrm{Cu}$ or $\mathrm{Au}$ as normal metal [4]-[7]. While Ti/Au bilayers may suffer aging problems [8], $\mathrm{Mo} / \mathrm{Au}$ bilayers display excellent chemical stability to stand the required conditions for detectors in spacecrafts [9]. However, deposition of Mo thin films with suitable and reproducible functional properties $\left(\mathrm{T}_{\mathrm{c}}\right.$, resistivity) usually requires temperatures above $500^{\circ} \mathrm{C}[10]-[17]$. These high temperatures may affect the quality of the interface [10]. Even more, they add difficulties to the prefabrication of heat sinking and electrical interconnection layers.

We recently demonstrated the capability to fabricate high quality Mo/Au bilayers at room temperature by RF magnetron sputtering [18]. Here we report a detailed structural characterization of bilayers and the analysis of the factors determining their performances and reproducibility.

\section{EXPERIMENTAL}

Mo thin films were deposited by Radio Frequency (RF) magnetron sputtering, at room temperature in an Ultra High Vacuum (UHV) system with a base pressure of $\sim 10^{-9}$ mbar. The Ar working pressure was $4 \times 10^{-3}$ mbar and the power was 210 $\mathrm{W}$. These deposition conditions give rise to a growth rate of 0.85 $\mathrm{nm} / \mathrm{s}$. The $\mathrm{Au}$ and $\mathrm{Cu}$ layers were deposited in the same UHV system by DC triode sputtering. Typical growth conditions for the $\mathrm{Au}$ and $\mathrm{Cu}$ layers were a target potential of $-1.0 \mathrm{kV}$ and a plasma-confining magnetic field of a few Oe. Typical deposition rates were of the order of $0.05 \mathrm{~nm} / \mathrm{s}(\mathrm{Cu})$ and $0.18 \mathrm{~nm} / \mathrm{s}(\mathrm{Au})$. The cathode-substrate distance was $8 \mathrm{~cm}$. In some of the bilayers Au was evaporated by electron beam in a different system. The growth rate for the evaporated gold films was $0.2 \mathrm{~nm} / \mathrm{s}$.

All samples were deposited on specially designed substrates: a $\mathrm{Si}_{3} \mathrm{~N}_{4}$ layer (200 nm-thick) was deposited by LPCVD on a $100 \mathrm{~nm}$ thick $\mathrm{SiO}_{2}$ layer thermally grown on a Si substrate and two $\mathrm{Si}_{3} \mathrm{~N}_{4}$ membranes $\left(400 \mu \mathrm{m}^{2}\right)$ were formed in it by etching the underneath $\mathrm{Si}$.

X-ray diffraction (XRD) was employed to study the crystal structure, preferential orientations and residual stress of the layers; $\theta-2 \theta$ scans at different angle $\Psi$ between the normal to the film and the normal to the diffraction planes were performed using a Bruker-AXS diffractometer with a GADDS bidimensional detector, model D8 Advance. 
Atomic Force Microscopy (AFM) was performed with a commercial microscope (Agilent 4500) as a tool to characterize surface topography and grain size of the films and bilayers; the data were analysed with the software Mountains, from Digital Surf.

Transmission Electron Microscopy (TEM) bright field images for grain size and layer thickness measurements were obtained using a Jeol 2100 working at $200 \mathrm{kV}$. High resolution characterization was carried out in a Jeol J2010F scanning TEM microscope, with a hot field emission gun, operating at $200 \mathrm{keV}$. Thin foils were prepared in cross section geometry (XTEM) by mechanical flat polishing down to $25 \mu \mathrm{m}$ and final Ar+ bombardment at $\mathrm{V}=4 \mathrm{kV}$ with an incident angle of $7^{\circ}$ using a PIPS-Gatan equipment.

Some of the Mo/Au bilayers were patterned by standard optical photolithography with a two-step wet chemical etching process. Firstly, the Au layer is etched in a KI/I solution through a photoresist mask. After mask removal by acetone, the Mo layer is etched in a Phosphoric/Acetic/Nitric acid solution which is selective to $\mathrm{Au}$. This procedure generates a gold layer overhanging the Mo edges and guarantees no parallel conduction through uncovered Mo [10].

The temperature dependence of the resistance was measured for patterned and unpatterned samples using the four-point resistance method; for layers with $T_{c}$ above $350 \mathrm{mK}$ a commercial PPMS system from Quantum Design was used; bilayers with lower $T_{c}$ were measured in a commercial dilution refrigerator (Kelvinox MX40, from Oxford Instruments) with base temperature $30 \mathrm{mK}$, and an AVS 47 resistance bridge. A LabView program was developed to control the power supplied to the mixing chamber at the dilution refrigerator. This program allows sweeping the temperature as slow as $1.7 \mathrm{mK}$ per hour; thus, very sharp superconducting transitions can be characterized. The transition is fitted to an exponential close to $R=0$ followed by a straight line at higher temperature, and $T_{c}$ is defined as the temperature where both fits intercept.

\section{RESULTS AND DISCUSSION}

\section{A. Mo Layers}

The XRD $\theta-2 \theta$ scans reveal that Mo thin films display a high degree of texture, with the (110) planes parallel to the growth direction. The residual stress has been analysed by the so-called $\sin ^{2} \Psi$ technique [19], consisting on measuring $\theta-2 \theta$ scans for different angles $\Psi$. Monitorization of the interplanar distance $\mathrm{d}$ for a certain reflection-obtained from the $\theta-2 \theta$ peak position at a given $\Psi$-as a function of $\sin ^{2} \Psi$ provides information on the stress of the film. Fig. 1 reveals such a plot for a $50 \mathrm{~nm}$ thick Mo film, using its (110) reflection. The linear dependence of $d$ on $\sin ^{2} \Psi$ reveals a biaxial stress in the plane, and the negative slope indicates that this stress is compressive. Using tabulated elastic constants for Mo $(\mathrm{E}=275 \mathrm{GPa}, \mathrm{v}=0.31)$ and the slope on Fig. 1, the stress may be estimated of the order of -1 GPa.

AFM and TEM (Fig. 4) images reveal the usual columnar growth of Mo. The distribution of grain sizes is important, and the average value depends on growth conditions, being typically of the order of $25-40 \mathrm{~nm}$. The films are quite flat: the typical

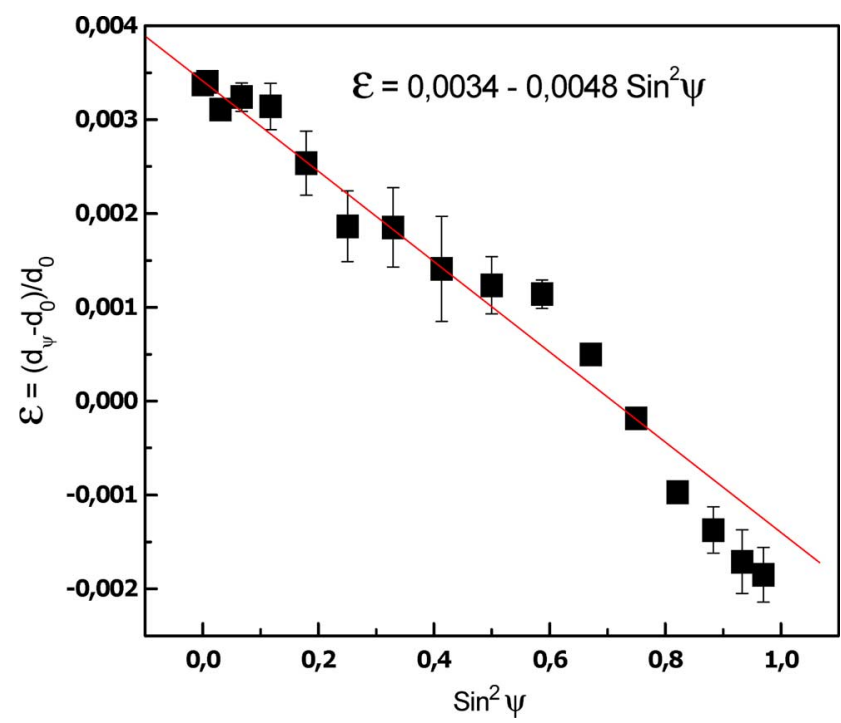

Fig. 1. Residual stress analysis of a Mo film $(50 \mathrm{~nm})$ The linear behavior of the strain $\varepsilon$ (where $\mathrm{d}_{\Psi}$ and $\mathrm{d}_{0}$ are respectively the (110) interplanar distance at an angle $\psi$ and its bulk value) as a function of $\sin ^{2} \Psi$ reveals a biaxial stress; the negative slope is indicative of compressive stress. The error bars correspond to the error in the peak position $(2 \theta)$ obtained from the peak fit.

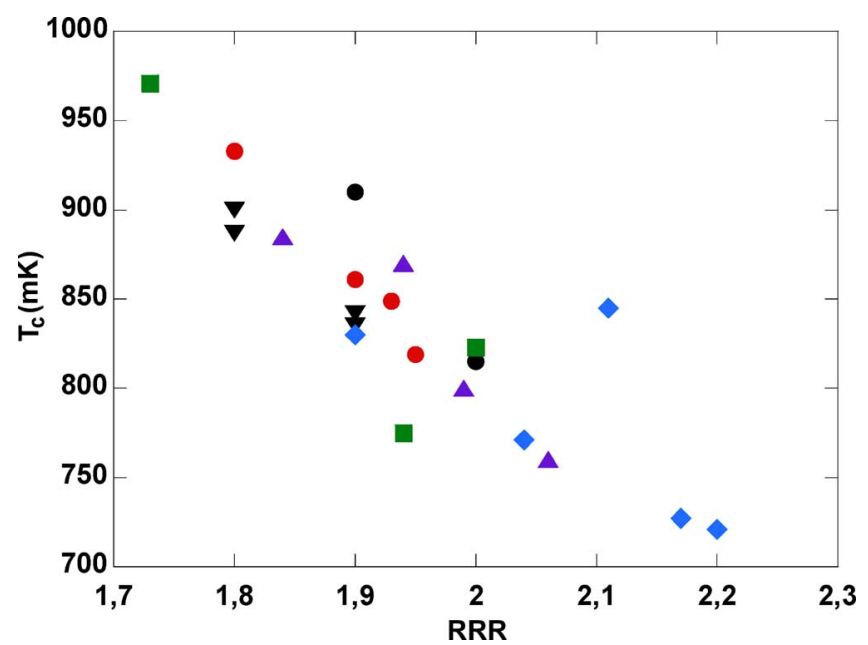

Fig. 2. Superconducting critical temperatures of bare Mo thin films $(50 \mathrm{~nm})$ obtained at deposition conditions close to optimal; each type of symbol stands for a series of samples grown testing a certain parameter (Ar pressure, target potential, RF power, substrate surface cleaning) while keeping the other constant). A clear correlation with RRR is observed.

average roughness over $1 \mu \mathrm{m}^{2}$ for a film $50 \mathrm{~nm}$ thick is $0.3 \mathrm{~nm}$, only slightly above surface roughness.

Bare Mo thin films display $T_{c}$ values between 700 and 900 $\mathrm{mK}$, close to the bulk $\mathrm{T}_{\mathrm{c}}=910 \mathrm{mK}$. We have identified two crucial factors affecting $T_{c}$ and its reproducibility on bare Mo layers: the Mo contamination in unprotected films, and their grain size. Fig. 2 displays the $T_{c}$ values of bare, unpatterned Mo films (50 $\mathrm{nm}$ thick), grown at slightly different conditions (Ar pressure, RF power, target potential). Clearly, $\mathrm{T}_{\mathrm{c}}$ correlates with RRR (Residual Resistance Ratio, measured between 300 $\mathrm{K}$ and $4.2 \mathrm{~K}$ ), which in turn depends on grain size. Therefore, controlling the deposition parameters, as well as the substrate surface, is crucial. Doing so, a series of 5 independent samples of $50 \mathrm{~nm}$ Mo with a protective layer of $15 \mathrm{~nm}$ Au deposited in 
TABLE I

RESISTIVITY AT 2 K AND RRR FOR NORMAL METAL FILMS (150 nm) GROWN AT DIFFERENT CONDITIONS

\begin{tabular}{|c|c|c|c|}
\hline Metal layer & $\rho(2 K)(n \Omega m)$ & RRR & \\
\hline Au sputtering & $61 @-1 \mathrm{kV}$ & 1.55 & (a) $-1 \mathrm{kV}$ \\
\hline & $102 @-2 \mathrm{kV}$ & 1.3 & @- $-2 \mathrm{kV}$ \\
\hline Au e-beam & 2.9 & 8.8 & \\
\hline $\mathrm{Cu}$ sputtering & $\begin{array}{l}12.6 @-1 \mathrm{kV} \\
20.8 @-2 \mathrm{kV}\end{array}$ & $\begin{array}{l}2.8 \\
4.8\end{array}$ & $\begin{array}{l}\text { @) }-1 \mathrm{kV} \\
\text { @- }-2 \mathrm{kV}\end{array}$ \\
\hline
\end{tabular}

situ was fabricated. The $T_{c}$ of all samples range between 675 and $705 \mathrm{mK}$, with RRR $=1.90$, hence showing an excellent improvement of reproducibility. The lowering of $T_{c}$ is due to the proximity effect.

We must point out that the decrease of Tc with increasing RRR displayed on Fig. 2 is opposite to the behavior commonly observed in Mo [17] and in most superconductors, although it is not unique. We are currently further investigating it.

\section{B. Metal Layers}

Au films deposited either by sputtering or e-beam are partially textured, with the (111) planes along the growth direction. A loss of texture is observed for thicker films, especially for those grown by e-beam. Residual stress analyses reveal a weak tensile stress of these films, of the order of $70 \mathrm{MPa}$.

The most crucial difference between Au films deposited by sputtering and e-beam is the resistivity. As shown on Table I, sputtered films display much higher resistivity values, which would result in a too high resistance of the device in the normal state, being thus unsuitable for TES operation. High resistivity values are commonly observed in sputtered gold films, and have been ascribed to high grain boundary scattering of the conduction electrons due to the small crystallite sizes [20]-[22]; it has also been suggested that Ar impurities might have a contribution [11].

$\mathrm{Cu}$ films deposited by sputtering are fully polycrystalline, with no preferential orientation along the growth direction. The crystal quality of these films is sensibly poorer than for $\mathrm{Au}$ films; in accordance, AFM images reveal $\mathrm{Cu}$ films rougher in the nanoscale than Au films. Residual stress measurements on $\mathrm{Cu}$ films indicate that they have a significant tensile stress $(\sim 400$ MPa). However, resistivity and RRR values, also displayed on Table I, are between the two types of Au films, and allow fabrication of devices with a resistance within the acceptable range for TES operation.

\section{Bilayers}

We have fabricated and characterized $\mathrm{Mo} / \mathrm{Cu}$ and $\mathrm{Mo} / \mathrm{Au}$ bilayers. $\mathrm{Mo} / \mathrm{Cu}$ bilayers have been grown in-situ by sputtering; as discussed above, Mo/Au bilayers require Au to be deposited by e-beam, in order to meet the resistance values requirements for TES. Since e-beam deposition is carried out in another UHV chamber, in order to avoid exposure of the Mo surface to air, and its eventual contamination, the Mo film was pre-coated and protected by a $15 \mathrm{~nm}$ thick Au layer deposited in-situ by sputtering. This thickness would guarantee a continuous coverage of the Mo film and would provide a perfect seed for the further growth of a thicker Au layer by e-beam deposition, which we

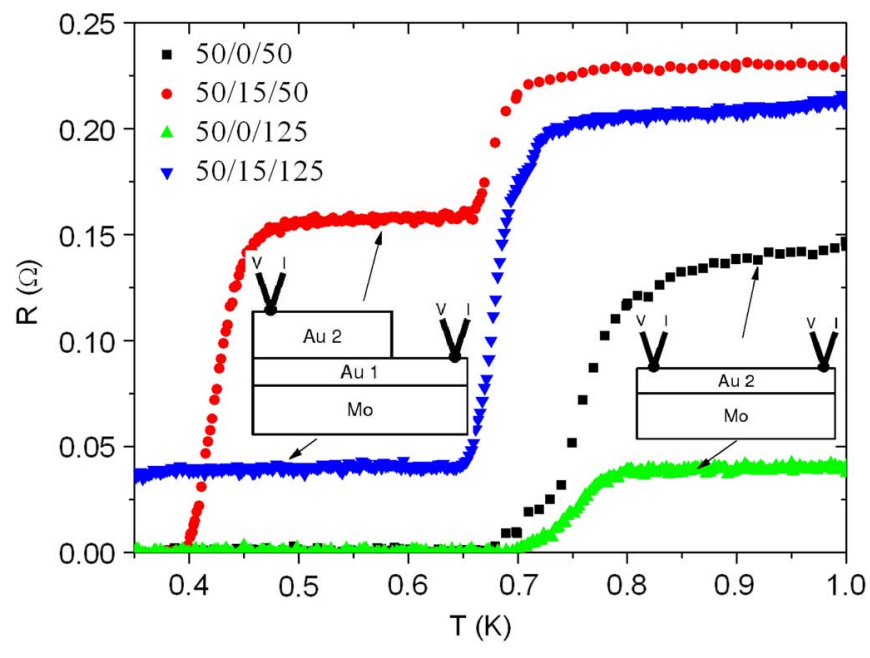

Fig. 3. Resistance behavior and transitions of unpatterned $\mathrm{Mo} / \mathrm{A} \mathbf{u}_{1} / \mathrm{Au}_{2}$ bilayers. The insets show the electrical configurations used to measure samples with and without a thin layer $\mathrm{Au}_{1}$ to protect $\mathrm{Mo}$, as described in text.

will call $\mathrm{Au}_{2}$, while we will refer to the thin sputtered layer as $\mathrm{Au}_{1}$.

In Fig. 3 we analyse the effect of the existence of $A u_{1}$ on the proximity effect, by comparing the $\mathrm{R}(\mathrm{T})$ curves for bilayers with and without $\mathrm{Au}_{1}$, for two different thicknesses of $\mathrm{Au}_{2}$. It may be observed that the two bilayers without $\mathrm{Au}_{1}$ display a single transition at roughly the critical temperature of bare Mo films, indicating that these bilayers are not affected by the presence of the $\mathrm{Au}_{2}$ layer. The 50/15/50 nm structure displays two transitions: the higher temperature one corresponds to the $\mathrm{Mo} / \mathrm{Au}_{1}$ bilayer (we have mentioned above that $50 \mathrm{~nm}$ Mo layers covered with $15 \mathrm{~nm}$ Au by sputtering display $\mathrm{T}_{\mathrm{c}}=690 \mathrm{mK}$ ); the lower temperature transition, leading to $\mathrm{R}=0$, is ascribed to the whole $\mathrm{Mo} / \mathrm{Au}_{1} / \mathrm{Au}_{2}$ structure, i.e. due to the proximity effect of layer $\mathrm{Au}_{2}$. Finally, the 50/15/125 nm structure displays only the higher temperature transition of $\mathrm{Mo} / \mathrm{Au}_{1}$ : the lower temperature transition may not be observed because it is expected to take place below $350 \mathrm{mK}$, the lowest temperature attained in the experiment.

These results clearly indicate that the proximity effect of $\mathrm{Au}$ is suppressed when Mo films are exposed to air. This fact is likely due to surface contamination of the superconductor, most likely surface oxidation. This effect will be further investigated using depth profile XPS measurements.

In view of the above results, all the Mo/Au bilayers studied in the following are $\mathrm{Mo} / \mathrm{Au}_{1} / \mathrm{Au}_{2}$, with $\mathrm{Mo}$ and $\mathrm{Au}_{1}$ fixed respectively to 50 and $15 \mathrm{~nm}$, and varying $\mathrm{Au}_{2}$.

Fig. 4 displays a cross section TEM image of a Mo/Au bilayer. A sharp and flat interface is observed between Mo and $\mathrm{Au}$, as a result of the Mo flatness and the absence of interdiffusion. $\mathrm{Mo} / \mathrm{Cu}$ bilayers display similar interfaces.

In-situ measurements of stress during Au growth by sputtering on Mo reveal that coalescence of Au takes place for very low thickness, around $2 \mathrm{~nm}$. Therefore, a $15 \mathrm{~nm}$-thick layer must be continuous across the film. However, as Fig. 4 reveals, it is difficult to appreciate the interfaces between the Au layers grown by sputtering and by e-beam; this is likely due to the fact that the sputtered Au film acts as a seed for the growth by 


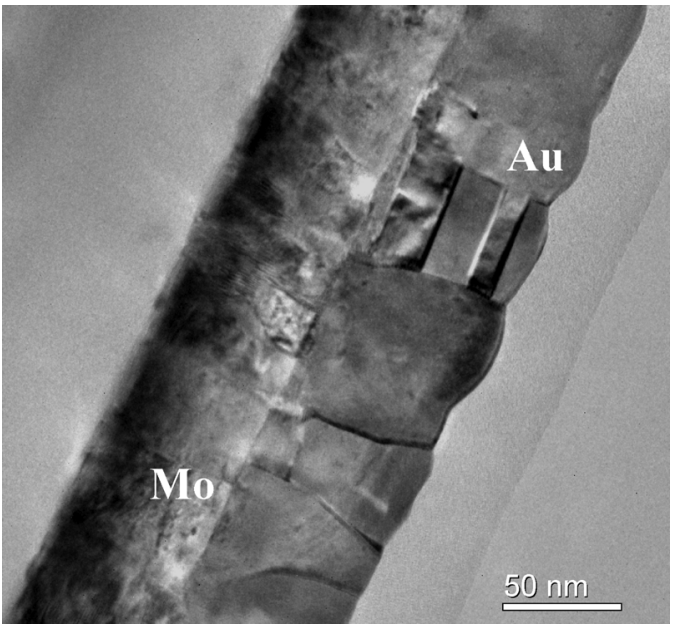

Fig. 4. Bright field cross-section TEM image of a bilayer constituted by $50 \mathrm{~nm}$ Mo, $15 \mathrm{~nm}$ Au deposited by sputtering and $50 \mathrm{~nm}$ Au deposited by e-beam.

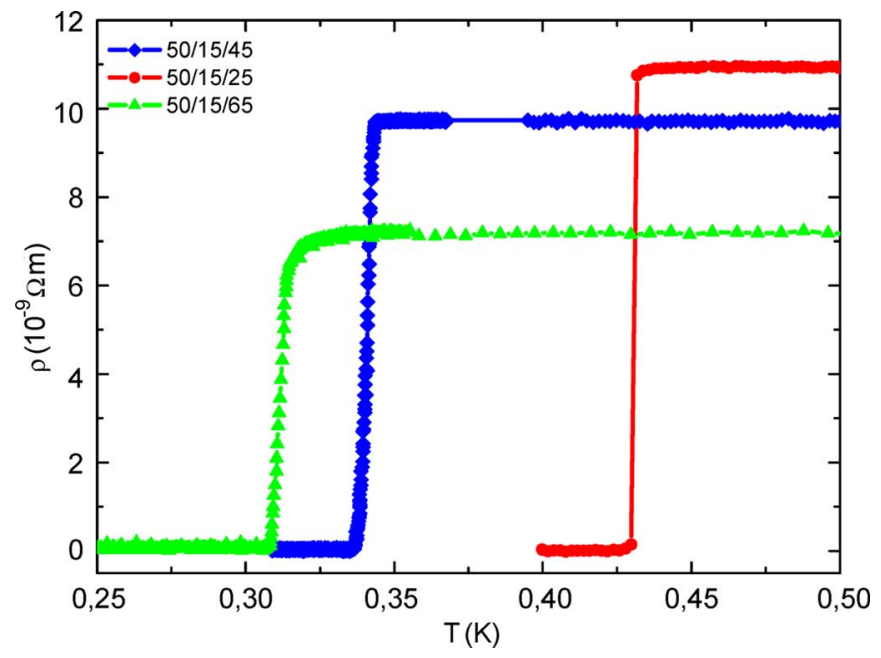

Fig. 5. Superconducting transitions of several patterned $\mathrm{Mo} / \mathrm{A} \mathbf{u}_{1} / \mathrm{Au}_{2}$ bilayers.

e-beam, and in most cases this results in the absence of contrast by TEM at the interface. We have observed that Au sputtered films grown on Mo films reproduce the columnar grains of Mo, whereas Au grown by e-beam usually displays twin boundaries parallel to the interface, as seen on Fig. 4. More detailed analyses of these bilayers are required in order to try to discern the details of the morphology of both Au layers.

We have performed residual stress analyses of the Mo and metal layers in the bilayers. It turns out that the stress state of Mo does not change significantly due to the presence of the normal metal layers. In fact, the stress values for $\mathrm{Mo}, \mathrm{Au}$ and $\mathrm{Cu}$ do not change when changing the $\mathrm{Au}$ or $\mathrm{Cu}$ thickness.

Fig. 5 displays the superconducting transitions of several patterned Mo/Au bilayers, with the intermediate $\mathrm{Au}_{1}$ layer. The sharpness of the transitions, between 1 and $5 \mathrm{mK}$, is worth emphasizing. As expected, $T_{C}$ is reduced when increasing the $\mathrm{Au}$ thickness; a similar effect has been observed for $\mathrm{Mo} / \mathrm{Cu}$ bilayers.

\section{CONCLUSIONS}

We have studied the effect of deposition conditions on the morphology, resistivity and critical temperature, and their reproducibility, for Mo-based bilayers. We have found that it is essential to prevent the exposure of Mo to atmosphere, and to control the grain size.

We have managed to obtain bilayers with good reproducibility and very narrow transitions. Further developments include in-situ growth of Au by e-beam and studies of the eventual effect of stress on the $T_{c}$ of the bilayers.

\section{ACKNOWLEDGMENT}

This work has been carried out within the EURECA (European-Japanese microcalorimeter Array) consortium. The authors thank helpful discussions with people from the Space Research Organization of the Netherlands, specially P. de Korte, M. Bruijn, J. van de Kuur and L. Gottardi. RGA and JS thank MEC for their Juan de la Cierva and Ramon y Cajal contracts, respectively. OG and MPB thank MEC for their fellowship.

\section{REFERENCES}

[1] J. A. Chervenak et al., "Fabrication of transition edge sensor X-ray microcalorimeters for constellation-X," Nucl. Instr. Meth. A, vol. 520, p. $460,2004$.

[2] P. A. J. de Korte et al., "EURECA: European-Japanese microcalorimeter array," J. Low Temp. Phys., vol. 151, p. 733, 2008.

[3] J. M. Martinis, G. C. Hilton, K. D. Irwin, and D. A. Wollman, "Calculation of Tc in a normal-superconductor bilayer using the microscopic-based Usadel theory," Nucl. Instr. and Meth. A, vol. 444, p. 23, 2000.

[4] A. L. Woodcraft et al., "Electrical and optical measurements on the first SCUBA-2 prototype 1280 pixel submillimeter superconducting bolometer array," Rev. Sci. Instrum., vol. 78, p. 024502, 2007.

[5] H. F. C. Hoevers et al., "Comparative study of TiAu-based TES microcalorimeters with different geometries," J. Low Temp. Phys., vol. 151, p. 94, 2008.

[6] J. N. Ullom et al., "Optimized transition-edge X-ray microcalorimeters with $2.4 \mathrm{eV}$ energy resolution at $5.9 \mathrm{keV}$," Appl. Phys. Lett., vol. 87, p. $194103,2005$.

[7] M. A. Lindeman et al., "Characterization and reduction of noise in Mo/Au transition edge sensors," Nucl. Instr. Meth. A, vol. 520, p. 348, 2004.

[8] A. Luukanen, H. Sipilä, K. Kinnunen, A. Nuottajärvi, and J. Pekola, "Transition-edge microcalorimeters for X-ray space applications," Physica B, vol. 284-288, p. 2133, 2000.

[9] , T. B. Massalski, Ed., Binary Alloy Phase Diagrams. Materials Park, OH: ASM International, 1990, vol. 1.

[10] T. C. Chen, F. M. Finkbeiner, A. Bier, and B. DiCamillo, "Molybdenum-gold proximity bilayers as transition edge sensors for microcalorimeters and bolometers," Supercond. Sci. Technol., vol. 12, p. $840,1999$.

[11] N. Tralshawala et al., "Fabrication of Mo/au transition-edge sensors for X-ray spectrometry," IEEE Trans. Applied Supercond., vol. 11, p. 755, 2001.

[12] E. Monticone et al., "Structural and electrical characterisation of Mo films for transition-edge sensors," Physica C, vol. 372-376, p. 440, 2002.

[13] J. E. Olsen et al., "Study of a Mo-Au TES deposited directly on a freestanding membrane," Nucl. Instr. and Meth. A, vol. 520, p. 296, 2004.

[14] M. Yun et al., "Fabrication of superconducting transition edge sensor based on Mo and Au bilayers," Nucl. Instr. and Meth. A, vol. 559, p. 462, 2006.

[15] A. N. Vystavkin, S. A. Kovtonyuk, and A. G. Kovalenko, "Experimental study of superconducting transition in a molybdenum-copper thin film structure showing the proximity phenomenon and the estimation of the sensitivity of TES bolometers on the basis of such a structure," Nucl. Instr. Meth. A, vol. 520, p. 289, 2004.

[16] R. Boucher et al., "Structural and electrical properties of AuPd/Mo bi-layer films for transition edge sensors," Supercond. Sci. Technol., vol. 19 , p. 138,2006 
[17] J. A. Chervenak et al., "Materials development for auxiliary components for large compact Mo/Au TES arrays," J. Low Temp. Phys., vol. 151, p. $255,2008$.

[18] R. González-Arrabal et al., "Mo/Au bilayers deposited by sputtering at room temperature for transition edge sensors fabrication," J. Low Temp. Phys., vol. 151, p. 239, 2008.

[19] B. D. Cullity and S. R. Stock, Elements of X-Ray Diffraction. Princeton, NJ: , 2001, Ed. Prentice Hall.
[20] P. A. B. Toombs and P. Bennet, "Electrical resistance of thin triodesputtered gold films," J. Appl. Phys., vol. 39, p. 2948, 1968.

[21] A. G. Blachman, "Stress and resistivity control in sputtered Molybdenum films and comparison with sputtered Gold," Metallurgical Trans., vol. 2, p. 699, 1971.

[22] C. Markert, D. Lutzenkirchen-Hecht, and R. Frahm, "Structural and electrical properties of thin d.c. magnetron-sputtered gold films deposited on float glass," Surf. Interface Anal., vol. 38, p. 715, 2006. 